Recepción: 16 / 01 / 2018

Aceptación: 28 / 02 / 2018

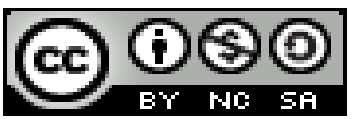

Ciencias de la salud

Publicación: 21 / 04 / 2018

Artículo de Revisión

\title{
Caries dental en niños de 6-12. Indicadores epidemiológicos
}

\section{Dental caries in children aged 6-12. Epidemiological indicators}

\section{Cárie dentária em crianças entre 6 e 12 anos. Indicadores epidemiológicos}

\author{
Socorro E. Zavarce-Pérez ${ }^{\mathrm{I}}$ \\ socorro.zarvace@gmail.com \\ Alcira M. Alvarado-Solórzano ${ }^{\text {II }}$ \\ alciraas@gmail.com \\ Betty E. Guanotoa-Lincango III \\ betty.guanotoa@gmail.com
}

Lourdes A. Rivadeneira-Proaño ${ }^{\text {IV }}$ lourdes.rivadeneria@gmail.com

Correspondencia: alciraas@gmail.com

\footnotetext{
I Especialista en Odontopediatría, Doctora en Ciencias de la Educación, Docente de la Universidad de Carabobo, Venezuela.
II Magister en Gerencia y Auditoria de Servicios de Salud Bucal, Odontóloga, Residente del post-grado de Odontopediatría de la Universidad de Carabobo, Docente en la Universidad Laica Eloy Alfaro de Manabí, Manta, Ecuador.

III Odontóloga, Ejercicio privado, Residente del post-grado de Odontopediatría de la Universidad de Carabobo. Venezuela.

Iv Odontóloga, Diploma Superior en Odontología Integral, Residente del post-grado de Odontopediatría de la Universidad de Carabobo. Venezuela.
} 


\title{
Resumen
}

El conocimiento de indicadores epidemiológicos de las caries dentales, resulta necesario debido a lo extendido que se observa este problema de salud entre la población de todas las edades. Se realizó una investigación de tipo descriptivo transversal, acerca de aspectos epidemiológicos de las caries dentales en 172 pacientes de 6 - 14 años. El estudio reveló la elevada prevalencia de dientes permanentes cariados, podía explicarse por la existencia de estilos de vida inadecuados, bajos ingresos económicos, dieta cariogénica, deficiente higiene bucal y antecedentes de caries dental, entre otros factores.

Palabras clave: niños; caries dental; indicadores epidemiológicos; factores de riesgo.

\begin{abstract}
The knowledge of epidemiological indicators of dental caries is necessary due to the widespread observed this health problem among the population of all ages. A cross-sectional descriptive research was carried out on the epidemiological aspects of dental caries in 172 patients aged 6 14 years. The study revealed the high prevalence of decayed permanent teeth, which could be explained by the existence of inadequate lifestyles, low income, cariogenic diet, poor oral hygiene and a history of dental caries, among other factors.
\end{abstract}

Keywords: children; dental caries; epidemiological indicators; risk factor's.

\section{Resumo}

O conhecimento dos indicadores epidemiológicos da cárie dentária é necessário devido à disseminação observada deste problema de saúde entre a população de todas as idades. Uma pesquisa descritiva transversal foi realizada sobre os aspectos epidemiológicos da cárie dentária em 172 pacientes com idade entre 6 e 14 anos. O estudo revelou a alta prevalência de dentes permanentes cariados, o que poderia ser explicado pela existência de estilos de vida inadequados, baixa renda, dieta cariogênica, má higiene bucal e história de cárie dentária, entre outros fatores.

Palavras chave: crianças; cárie; indicadores epidemiológicos; fatores de risco.

\section{Introducción}

La caries dental es un problema de salud pública que afecta a la población en cualquier parte del mundo, y por consecuencia a los ecuatorianos. 
La importancia clínica y el coste social de la caries dental en niños en edad escolar son enormes; esta enfermedad es considerada un problema de salud pública debido a su alta prevalencia e incidencia. En países industrializados, se ha observado una disminución de la prevalencia de caries en décadas pasadas. Algunos datos epidemiológicos recientes sobre caries dental muestran una importante mejoría en la salud bucal de niños de Latinoamérica y del Caribe, aunque todavía hay considerables necesidades preventivas y de tratamiento rehabilitador que no han sido satisfechas. El uso generalizado de fluoruros proveniente de diversas fuentes parece ser el principal factor en la reducción de la prevalencia de caries. Otras posibles explicaciones son la instauración de programas de salud bucal ${ }^{8}$ y cambios en el criterio diagnóstico. El grupo de trabajo conjunto FDI/WHO también señaló que los servicios dentales han contribuido a la disminución de caries dental como resultado de mayor disponibilidad de recursos dentales, la provisión de tratamientos preventivos y una cultura dental más favorable, derivada de la educación en salud. ${ }^{1}$

La caries dental ha sido descrita como una enfermedad multifactorial relacionada con la dieta, bacterias intraorales, composición de la saliva y otros factores. ${ }^{2}$

Bajo otras circunstancias, puede considerarse como una enfermedad infecciosa causada por la flora normal de la cavidad oral (Peres SH de Carvalho Sales. Perfil epidemiologico de carie dentaria, em cidades fluoretadas e nao fluoretadas, na regiao centro- oeste do estado Sao Paulo. Bauru; s. n; 2001. p. 180). Como muchas enfermedades infecciosas, una masa crítica de bacterias cariogénica es un prerrequisito, y esta masa crítica puede obtenerse solo en presencia de sacarosa, un sustrato en el que la caries se desarrolla. ${ }^{3}$

Así la caries dental involucra la interacción en el tiempo de una superficie dental susceptible, las bacterias cariogénicas, y la disponibilidad de una fuente de carbohidratos fermentables, especialmente sacarosa. ${ }^{4}$

La infección bacteriana es necesaria, pero no suficiente para el desarrollo de la enfermedad; deben estar presente una serie de factores (factores de riesgo), para que se desarrolle la misma ${ }^{5-6-4}$ En el marco de las observaciones anteriores todos esos criterios sirvieron de argumentación para ejecutar este trabajo, teniendo en cuenta la importancia que reviste para el estomatólogo conocer 
el estado de salud-enfermedad de niños y niñas, incluidos los distintos factores que intervienen en la aparición de las caries.

\section{Metodología}

Se realizó un estudio descriptivo y transversal sobre epidemiología de las caries dentales en pacientes de 6 - 14 años, atendidos en la consulta de odontopediatria durante el primer trimestre del 2017.

La población de estudio (172), fue diseñada siguiendo un modelo estratificado, con selección aleatoria simple y utilizando como variables de estratificación la edad y el sexo. Para escoger a los pacientes, se tomó de la lista de la consulta diaria a los que presentaban dolor u otra causa que constituyera una urgencia odontológica.

Como indicadores epidemiológicos, se utilizó el CPO-D según grupos de edades; se consultó la historia clínica. Se examinó a los pacientes bajo la luz artificial de la unidad dental, con el instrumental de diagnóstico previamente esterilizado (espejo y explorador bucal y pinza para algodón) y el uso de la jeringa de aire y agua, sin obviar el correspondiente lavado de las manos antes y después de cada examen bucal.

Se calculó el índice de COP-D para estimar la prevalencia de caries, considerando toda la historia de la enfermedad tratada al incluir los dientes que estaban afectados por caries dental, los que habían sido obturados y aquellos que fueron extraídos o perdidos por esa causa.

Se aplicó una encuesta a los padres con respecto al nivel de conocimiento acerca de la higiene bucal.

\section{Resultados}

Como muestra la tabla 1 , el $66.1 \%$ de los niños examinados se encontraban afectados por caries dental, predominando el grupo de edades de 6 a 8 años. 
Tabla 1. Población afectada y no afectada por grupo de edades.

\begin{tabular}{|l|c|c|c|c|c|c|}
\hline \multirow{2}{*}{$\begin{array}{c}\text { Grupo de } \\
\text { edades }\end{array}$} & \multicolumn{2}{|c|}{ Afectados } & \multicolumn{2}{c|}{ No afectados } & \multicolumn{2}{c|}{ Total } \\
\cline { 2 - 7 } & No & $\%$ & No & $\%$ & No & $\%$ \\
\hline $6-8$ & 66 & 47.4 & 20 & 60.6 & 86 & 50.0 \\
\hline $9-11$ & 45 & 32.3 & 11 & 33.3 & 56 & 32.5 \\
\hline $12-14$ & 28 & 20.1 & 2 & 6.0 & 30 & 17.4 \\
\hline Total & $\mathbf{1 3 9}$ & $\mathbf{6 6 . 1}$ & $\mathbf{3 3}$ & $\mathbf{1 9 . 1}$ & $\mathbf{1 7 2}$ & $\mathbf{1 0 0}$ \\
\hline
\end{tabular}

Con respecto al índice de al índice de CPO -D por dicha enfermedad (tabla 2), se halló que fue de 1.0, con la mayor afectación en el grupo de edades de 12-14 años con un índice de 2.8.

Tabla 2. Indicadores epidemiológicos de COP-D según grupos de edades.

\begin{tabular}{|l|c|c|c|c|}
\hline $\begin{array}{l}\text { Grupo de } \\
\text { edades }\end{array}$ & $\begin{array}{c}\text { Dientes } \\
\text { cariados }\end{array}$ & $\begin{array}{c}\text { Dientes } \\
\text { obturados }\end{array}$ & $\begin{array}{c}\text { Dientes } \\
\text { perdidos }\end{array}$ & $\begin{array}{c}\text { Indice de } \\
\text { COP }\end{array}$ \\
\hline $6-8$ & $3(0.03)$ & $6(0.06)$ & $3(0.03)$ & 0.1 \\
\hline $9-11$ & $49(0.8)$ & $18(0.3)$ & $4(0.07)$ & 1.2 \\
\hline $12-14$ & $56(1.8)$ & $20(0.6)$ & $10(0.3)$ & 2.8 \\
\hline Total & $\mathbf{1 0 8 ( 0 . 6 )}$ & $\mathbf{4 4 ( 0 . 2 )}$ & $\mathbf{1 3}(\mathbf{0 . 0 7})$ & $\mathbf{1 . 0}$ \\
\hline
\end{tabular}

De los dientes permanentes, $63.0 \%$ estaban cariados, con predominio en grupos etarios de 11-12 años). Así mismo, se encontró que 5.5\% de las extracciones fueron indicadas a los 8 años y a los 9.

En la casuística de la tabla 3, se observa que el factor de riesgo que predomino fue la dieta cariogénica $(87.2 \%)$ seguido de la mala higiene bucal $84.3 \%$. 
Tabla 3. Factores de riesgo presentes.

\begin{tabular}{|l|c|c|}
\hline \multicolumn{1}{|c|}{$\begin{array}{c}\text { Factores de riesgo } \\
\text { presentes }\end{array}$} & No & \% \\
\hline $\begin{array}{l}\text { Experiencia de caries } \\
\text { anterior. }\end{array}$ & 44 & 25.5 \\
\hline Dieta cariogénica. & 150 & 87.2 \\
\hline Mala higiene bucal. & 145 & 84.3 \\
\hline Flujo salival escaso. & 56 & 32.5 \\
\hline Apiñamiento dentario & 72 & 41.8 \\
\hline Otros factores & 23 & 13.3 \\
\hline
\end{tabular}

Al valorar el nivel de conocimiento que poseían los padres, con respecto a la importancia de la higiene bucal como factor de riesgo para las caries dentales, el $51.1 \%$ fue adecuado y $48.8 \%$ inadecuado

Tabla 4. Nivel de conocimiento de los padres con respecto a la higiene bucal.

\begin{tabular}{|l|c|c|}
\hline Nivel de conocimiento & No & \% \\
\hline Adecuado & 88 & 51.1 \\
\hline No adecuado & 84 & 48.8 \\
\hline
\end{tabular}

\section{Discusión}

La caries dental es frecuente entre nuestros escolares, y continua siendo la afectación odontológica más frecuente entre toda la población menor de 14 años. ${ }^{7}$

Varios autores investigan acerca de la aparición de la caries dental durante los primeros años después de haber brotado la primera dentición, los cuales coinciden con nuestros resultados. ${ }^{8}$ 
El componente cariado es el que mayor aporte tuvo al índice de caries dental en ambas denticiones. Esto puede ser interpretado, como una necesidad de atención bucal para el tratamiento y prevención de lesiones cariosas en el grupo de niños. El índice de caries dental hallada en la investigación es similar a lo reportado en estudios previos realizados a nivel internacional y nacional ${ }^{9}$. Sin embargo, la prevalencia global de este estudio es inferior a lo reportado por Rodríguez et al. ${ }^{10}$ en un estudio realizado en niños institucionalizados en España.

El componente extraído es el que menor contribución tuvo al índice, lo cual podría ser explicado en parte por el escaso acceso a los servicios de salud por atención bucal, en otros casos, la severidad de las lesiones cariosas en el grupo de niños, no comprometen aun la pulpa dentaria y al no ser percibido como una emergencia no recibe tratamiento dental conservador.

La higiene es un factor de riesgo que influye de forma determinante en la aparición de esta infección de la cavidad bucal; constituye un punto importante en el que el aspecto educativo tiene un papel fundamental. Comienza desde la casa con la participación de los padres y debe continuar en la escuela donde maestros y auxiliares pedagógicas pueden ayudar a controlar este riesgo y así evitar que aparezcan las caries dentales. Numerosos autores confirman que la mala higiene bucal es un riesgo significativo en la caries dental, y que se encuentra relacionada con su prevalencia ${ }^{11-12}$. Diversos estudios confirman que una buena higiene bucal tiene un gran impacto en la futura salud dental, por lo que se deben cambiar los hábitos de higiene inadecuados para prevenir las caries. Se recomienda el cepillado dental; si se realiza solamente una vez al día contribuye a la mala higiene bucal, y la confirma como un factor de riesgo, pero si su uso es adecuado contribuye a su prevención, particularmente si se utilizan dentífricos fluorados. ${ }^{7}$

La experiencia anterior de caries es considerada como una variable clínica de importancia, ya que la afectación en edades tempranas conforma los nichos ecológicos que favorecen su aparición posterior, hecho que es estudiado por varios autores con resultados similares a los nuestros.$^{13-14}$

A pesar del elevado número de pacientes de todos los grupos etarios que requieren atención odontológica, es preciso considerar la posibilidad de estructurar y aplicar programas integrales (preventivos y curativos) en edades más tempranas de la vida, a fin de resguardar el futuro de la salud bucal mediante la realización de actividades, encaminadas a efectuar una labor de saneamiento en los niños de dichas edades, de manera que se incluyan aspectos como la 
colaboración de los padres en las medidas de auto-gestión, la inclusión de la salud bucal entre las prioridades de la salud general del individuo, el desarrollo de hábitos sanitarios positivos en los niños, la aplicación de criterios más afines para el tratamiento de lesiones cariosas y la identificación de pacientes con alto riesgo de presentar caries. Estas medidas perseguirían lograr un mayor impacto en la salud bucal y alcanzar las metas nacionales e internacionales de salud bucodental.

Se concluye, que la elevada prevalencia de dientes permanentes cariados, podía explicarse por la existencia de estilos de vida inadecuados, bajos ingresos económicos, dieta cariogénica, deficiente higiene bucal y antecedentes de caries dental, entre otros factores.

\section{Referencias bibliográficas}

1. Herrera Míriam del Socorro, Medina-Solis Carlo Eduardo, Maupomé Gerardo. Prevalencia de caries dental en escolares de 6-12 años de edad de León, Nicaragua. Gac Sanit . 2005 [citado 2017 Mayo 19]; 19(4): 302-306. Disponible en: http://scielo.isciii.es/scielo.php?script=sci_arttext\&pid=S0213-91112005000400006\&lng=es.

2. Colectivo de autores. Guías Prácticas de Estomatología. Cap. 1. Guías prácticas clínicas de caries dental. La Habana: Editorial Ciencias Médicas; 2003. pp. 23-34.

3. Felton González, et al. Características epidemiológicas de prevalencia da cárie dentaría na denticao decídua, em pacientes de nivel socio-económico médio-alto. Rev Paul Odontol 1986;8(1):2-6.

4. Hidalgo Gato- Fuentes Iliana, Duque de Estrada Riverón Johany, Pérez Quiñones José Alberto. La caries dental: Algunos de los factores relacionados con su formación en niños. Rev Cubana Estomatol. 2008 Mar [citado 2017 Mayo 19]; 45(1). Disponible en: http://scielo.sld.cu/scielo.php?script=sci_arttext\&pid=S0034-75072008000100004\&lng=es.

5. OPS. La salud en las Américas. Publicación Científica y Técnica. Edición 2002; I y II: 587

6. Erickson PR, Alderegia. Estimation of the caries- realted risk associated with formula. Pediatr Dent 1998;20(7):395-403. 
7. Luján Hernández L, Luján Hernández M, Sexto N Revista Científica Electrónica de las Ciencias Médicas en Cienfuegos. Medisur 2007;5(2) ISSN:1727-897X

8. Reisine ST, Psoter W. Socioeconomic status and select behaviour al determinants as risk factors for dental caries. J Dent Educ. 2001;65 (10):1009-16.

9. Cortés-Martinicorena FJ, Doria-Bajo A, Asenjo-Madoz MA, Sainz de Murieta-Iriarte I, Ramón-Torrel JM, Cuenca-Sala E. Prevalencia de caries y estado periodontal de los niños y adolescentes de Navarra (2002). RCOE. 2003; 8 (4): 381-390.

10. Rodríguez J, Rivera M, López JM, Uberos J, Peñalver MA, Muñoz A. Salud bucodental en menores institucionalizados de Granada. Bol SPAO. 2009; 3 (3): 74-95. Citado 20 febrero 2014. Disponible en: http://hera.ugr.es/tesisugr/18847572.pdf .

11. Pimienta FC, Marin JM. Prevalence of mutans ingerprinti in 93 members fron six Brazilian families. Pesqui Odontol Bras. 2001;15(3):181-6.

12. Gispert Abreus E, Rivero López A, Cantillo Estrada E. Relación entre el grado de infección por Streptococcus mutans y la posterior actividad cariogénica. Rev Cubana Estomatol.2000;37 (3):157-61.

13. Creedon MI, O`Mullane DM. Factors affecting caries levels amongst 5- year-old children in County Kerry, Ireland. Community Dent Health. 2001; 18:72-8.

14. De Barros SG, Alves AC, Purgliese LS. Contribution to the study of dental caries in 0 -30 mont-old infants. Pesqui Odontol Bras. 2001;15(3):215-2 